\title{
Sexual Orientation Change Efforts and VBP
}

\author{
Taryn Knox
}

\subsection{Introduction}

Although gay people still face discrimination, being gay has become increasingly accepted in the modern western world. In turn, sexual orientation change efforts (SOCE) — methods used to alter an individual's sexuality, predominantly from homosexual to heterosexual-are largely considered ethically unacceptable. But what if an individual wants SOCE and gives truly informed consent? This question raises a number of conflicting values. A central and unavoidable issue is how these conflicts of values should be resolved. This chapter focuses on whether the conflicts can be resolved by considering whether there are some values that, according to VBP, should not be taken into account.

The chapter explores this issue primarily through the story of 'Matthias' and his therapist 'Kirk' (not their real names). The adapted narrative describes Matthias, a gay man who questions his sexuality and is considering having SOCE. The story is extensively adapted from a case study reported by Andrew Kirby [1]. The overarching question posed by the case study is whether Matthias should have conversion therapy. This question makes explicit the cultural and other values underpinning the issues in a situation of this kind. However, this chapter focuses on which values are relevant for the purposes of decision-making in VBP.

Two notes on terminology. Firstly, the chapter uses the phrase 'SOCE' rather than the more widely known phrase 'conversion therapy'. While the two refer to the same thing, the chapter uses SOCE. This is because the word 'therapy' in 'conversion therapy' signifies that being gay is a disease or illness in need of therapeutic intervention. In contrast, SOCE remains neutral on this issue. Secondly, in the literature, the term heterosexism refers to prejudiced institutions and structures, and

\footnotetext{
T. Knox $(\bowtie)$

Bioethics Centre, University of Otago, Dunedin, New Zealand 
the term homophobia to prejudiced personal attitudes (rather than, as the term suggests, fear) [2]. I use homophobia as shorthand for both homophobic and heterosexual values. In the second part of this chapter, it is suggested that there may be values that do not align with homosexuality, yet are not prejudiced.

\title{
39.2 Case Narrative
}

\begin{abstract}
Matthias (not his real name) is a confident, attractive gay man in his mid-thirties who was raised in a conservative Christian home. He recalls having always been attracted to men rather than women and 'came out' to his parents in his early twenties. Despite some sadness that he had disappointed his parents by being gay, he has generally felt loved and supported by his family. After several short-term relationships, Matthias settled into a happy long-term relationship of 10 years with another man. Matthias enjoys an accomplished career and has a close circle of friends both in and out of the gay community. On entering therapy Matthias described a conflict between his homosexuality and a desire to have children of his own. This, combined with his Christian faith that does not support same-sex attraction, created an underlying gnawing angst that often made him question his gay identity and lifestyle [1].
\end{abstract}

Over a period of time, Matthias explored these issues with a therapist, Kirk (not his real name), who took a gay-affirmative perspective. While Matthias gained insight into how formative influences contribute to his core belief system and values, Matthias was unresponsive to the gay-affirmative approach. Matthias brings up the topic of SOCE, and asks Kirk whether he thinks it would be a good idea for himself (Matthias) to try it.

Kirk is somewhat taken aback, as he is himself gay and believes that Matthias could lead a happy and fulfilling life as a gay man. Kirk knows that SOCE is available in the city in which Matthias lives. Kirk is not focused on the issue of whether SOCE should be banned, but is more troubled by whether Matthias, in particular, should have SOCE. He also wonders whether he has an obligation to refer Matthias to someone who practices SOCE, even though he (Kirk) is personally offended by the practice.

After doing some research, Kirk finds that SOCE is not currently effective, and in many cases, it is harmful [2,3]. However, Kirk also reads that recent advances in neuroscience mean that in the near future, there may be SOCE that is both effective and safe [3]. If so, would his intuition that Matthias should not have SOCE still stand up to scrutiny? The issue of consent also crosses Kirk's mind. He knows that many instances of SOCE are either forced or coercive, and holds that any SOCE that is not truly consensual is unacceptable. Yet, Kirk thinks that Matthias may give his truly informed consent to SOCE. If SOCE were safe and effective, and if Matthias gave genuine informed consent, should Matthias have SOCE? And what should Kirk do if Matthias were to ask him to refer him to someone who practices SOCE? Night after night Kirk lies in bed considering these questions.

Kirk is not the only one suffering sleepless nights. Matthias also spends many long nights agonising over whether he should have SOCE. Matthias recognises that 
his values (his beliefs and desires) are somewhat conflicting. On the one hand, Matthias identifies as gay and is open to gay-affirmation therapy. He definitely values authenticity i.e. being true to his gay self. Matthias also has a deeply loving and respectful relationship with his partner Lachlan. It would be impossible to have SOCE and stay in a relationship with Lachlan. These points make Matthias think that he should not have SOCE. On the other hand, family is very important to Matthias. Not only does he want to have children of his own, he has continuing fears of disappointing his parents. Matthias acknowledges that his religious values are a strong factor causing his uneasiness about his sexuality, as his Christianity has become increasingly important to him.

Matthias recognises the conflict between, on the one hand, his identity as a gay man and wanting to stay in a relationship with Lachlan (which provide reasons not to have SOCE), and on the other hand his desire to have children of his own, please his family and retain his faith (which point in favour of SOCE). He racks his mind to come up with a way in which the conflict could be avoided. Matthias knows that a gay man can have children of his own. But what he really wants is to have the biological children of both himself and his partner, and it is currently impossible to have the biological children of both Lachlan and himself. The divergence between Matthias' desire to have children and his sexuality remains. Matthias also knows that there are gay-friendly churches in his city-that Christianity is compatible with homosexuality. However, Matthias does not entirely approve of these gay-friendly churches, and maintains that his personal Christianity is incompatible with homosexuality. If Matthias could accept that he can have his own biological children (though not children that are biologically related to both himself and Lachlan) and if he could accept a gay-friendly iteration of Christianity, then there would be no need for him to have SOCE. But Matthias cannot accept either of these things, and concludes that he holds values that are intractably incompatible. His sleepless nights continue.

While tossing and turning the following night, Matthias begins to think about how others may be impacted by his decision to have-or not have-SOCE. He knows that Lachlan would be devastated if he decided to go ahead with SOCE. But to what extent should Lachlan's perspective have an effect on Matthias' decision whether or not to have SOCE? Should he decline SOCE just to keep Lachlan happy? Matthias knows that some of his gay friends would be very angry with him if he were to have SOCE. They would say that doing so would diminish and disempower the already vulnerable gay community $[2,4]$. Matthias is sympathetic to this argument to some degree, but wonders whether the needs of the gay community should override his individual desire to have SOCE (if this is indeed what he decides he wants).

Just before drifting into a fitful sleep, Matthias begins to think about Kirk, his therapist. Matthias knows that Kirk is gay, and that he is a strong believer in gayaffirmative therapy. Matthias has developed a meaningful relationship with Kirk, and worries about how Kirk would respond if he (Matthias) were to go ahead with SOCE. But once again, should Matthias do what is right for him or that which is right for others? 
Matthias takes the courageous step to discuss some of these issues with Kirk. Kirk mentions that Matthias' gnawing angst concerning his sexuality is not really his own view, but may stem from an internalisation of homophobic sentiment in society. Kirk and Matthias discuss that rather than Matthias having SOCE, society ought to be changing the dubious social norms [2]. Together, Matthias and Kirk explore whether Matthias has had negative experiences relating to his sexuality in family, social, educational or vocational contexts, and has incorporated this antigay sentiment into his own belief systems. Matthias denies that his views are caused by an internalisation of external anti-gay sentiment-he maintains that they are truly his own views, and that his desire for SOCE are based on his genuine values. (Without relating it to Matthias, Kirk worries that if he were to refer Matthias to someone practicing SOCE, then he might even be compared to a doctor who gives his or her patient in an abusive relationship Valium, rather than dealing with the underlying problem.)

Matthias then points out that New Zealand-the country in which he lives-is relatively gay-friendly, while recognising that gay people in New Zealand undoubtedly experience some stigmatisation. Matthias goes on to say that it is not the case that he has internalised homophobic views, but it may be the case that he has internalised gay-friendly views. That is, he wonders whether the predominantly gayfriendly values of New Zealand society have actively discouraged him from having SOCE. Does he really want SOCE, but is worried about the social consequences of doing so? Will his gay friends be angry with him? Will he be accused of setting the gay-rights movement back 20 years?

Should Matthias have SOCE? If asked, is Kirk obliged to refer Matthias to someone who practices SOCE? The latter part of this chapter interrogates the conflict of cultural values using the insights of Fulford's 'Values-Based Practice' (VBP).

\subsection{Commentary}

Matthias's values - his needs, preferences, strengths and aspirations - are of fundamental importance (principle four). Some of Matthias's internal values conflict. He identifies as gay and wants to stay in his relationship with Luke. Yet, he also wants biological children of his own and his personal Christianity is incompatible with being gay. Given the importance of informed consent in health care (here, 'health care' is interpreted broadly to include SOCE), it is clear that SOCE should only be given to Matthias if, after balancing his internal conflicting values, he gives his genuine and informed consent. If Matthias refuses to give consent, the issue largely dissolves. Matthias will not have SOCE, which would align with the values of Kirk, Lachlan and the gay community. The difficulty arises if Matthias decides he does want SOCE, as there is a conflict of values between himself and others.

Rather than focusing on the internal conflict between some of Matthias's values, this commentary focuses on a specific external conflict-the conflict between Matthias's religious values (which support SOCE) and the values of a gay-friendly 
society (which suggest that having SOCE is not compatible with mutual respect for gay people). Other external conflicts, such as the conflict between Matthias's desire for SOCE and the values of his therapist (Kirk) and partner (Lachlan), are not directly considered.

The chapter considers it to be possible that Matthias's views are not internalised homophobic norms, but does not directly consider whether Matthias has, in fact, internalised anti-gay sentiment in society (see, for example, Cruz in [3]). This is because, even if Matthias's views are internalised anti-gay norms, many choices are 'profoundly influenced by social pressures ... but we wouldn't normally conclude that people are therefore non-autonomous [have not given genuine consent]' [3]. Hence, determining whether or not Matthias has internalised social norms does not necessarily change whether SOCE ought to be available to him. In short, the chapter proceeds on the basis that Matthias' views are not internalised homophobic norms, and even if they were internalised, it does not automatically mean that he should not have SOCE.

Diversity of values plays an integral role in VBP. VBP is clear that, in addition to Matthias's values, the values of other parties involved also need to be considered (principle five). Moreover, decisions are not made by determining the right outcome but by a process that balances multiple legitimately different perspectives. The word 'legitimately' plays two roles. Firstly, it emphasises that having differing values is not a bad thing. Secondly, it hints that there are some perspectives or values that are illegitimate. According to VBP, legitimate values are those that are consistent with mutual respect. Fulford gives the example of racism: racism is incompatible with respect for differences, and so racist values are precluded from VBP. Fulford also considers sexist, homophobic, classist, ageist and ableist values, along with values that are prejudiced towards religion, culture and language, to be incompatible with mutual respect [5].

Pickering (chapter 33, "Covert treatment in a cross-cultural setting") critiques VBP on the basis that the scope of legitimate values differences is the same as the scope of values differences, i.e. that there is no way of distinguishing between legitimate and illegitimate values. But VBP does provide a method for distinguishing between the two, namely, whether the values are compatible with mutual respect. Nonetheless, Fulford does not provide a justification for having mutual respect as the necessary and sufficient condition of a legitimate value. And why 'mutual respect' as opposed to simply 'respect'? Let's put these problems aside, and directly consider the case study in question.

Two problems develop regarding Matthias's situation. Firstly, homophobic values and values that are prejudiced towards religion are not consistent with mutual respect, and in turn are not VBP-able, i.e., cannot be accounted for in the decisionmaking process. Matthias's desire to have SOCE appears to be a homophobic value, and so is beyond the pale of VBP. It is not a legitimately different perspective and cannot be accounted for in the decision-making process. Given principle four (the patient-perspective principle), this seems very odd. Surely Matthias's values, including his religious values, ought to play a central role when making decisions that 
primarily, although not solely, impact him. Moreover, all VBP case studies concern the conflicts of values between the various stakeholders, rather than conflicting values held by an individual such as Matt. This suggests that Fulford did not intend VBP to have anything to say about such private decisions.

Secondly, the list of values that are not VBP-able may themselves conflict. For example, female genital mutilation (FGM) may not be compatible with respect for women, but not allowing FGM may be incompatible with respect for cultural values [6]. A comparable paradox arises in Matthias's case. Matthias's values that lead him to consider SOCE could be homophobic, and so incompatible with mutual respect for the gay community and, in turn, not VBP-able. Yet, limiting Matthias's religious freedom (by not allowing him to have SOCE, or not allowing Matthias's religious values to be factored into VBP) is also incompatible with mutual respect. Hence, values that limit religious freedom are not VBP-able, which suggests Matthias's religious values need to be accounted for in VBP.

A potential way of avoiding this paradox is to show that one or both of those values are consistent with mutual respect, and so can be factored into the decision-making process. Carrying out violent anti-gay hate crime is clearly inconsistent with mutual respect. However, Matthias's desire to have SOCE might be compatible with mutual respect if, for example, he thought that while he does not want to be gay, it is fine for other people to be gay. In the same vein, it might be inconsistent with mutual respect of religious groups to ban SOCE. However, taking a more moderate approach, such as requiring Matthias to enter into discussion about the acceptability of SOCE, is likely to be consistent with mutual respect.

VBP might deem all values that might loosely be considered homophobic (and racist, sexist, classist, ablest values and values that are prejudiced towards religion, culture and language) to be inconsistent with mutual respect and therefore not VBPable. For example, one can imagine that Matthias' parents had dreams of being grandparents. This is most easily achieved if Matthias is straight. The desire for grandchildren could be considered a homophobic value, although it is only homophobic in a benign way. That is, taken on its own, it is only very minimally prejudiced. If all values that are incompatible with mutual respect in a benign way are beyond the pale of VBP, then VBP would become very difficult to operationalise. Hence, it is likely that Fulford did not intend 'values incompatible with mutual respect' to have such a broad reach.

Principles six and seven require those practising VBP to look deeply at the exact values involved. Based on this, when considering whether a perspective is compatible with mutual respect, it appears that VPB requires us to consider the precise value or perspective involved. It would be easy to blithely label Matthias's desire to have SOCE as homophobic. However, VBP requires more, namely, to consider whether Matthias's particular view is consistent with mutual respect. Matthias's view-while he does not want to be gay, it is fine for other people to be gay-is, at least arguably, consistent with mutual respect. 
Of course, some people may consider Matthias's view to be incompatible with mutual respect, i.e. to be homophobic. They might point out that as the gay community has faced much oppression and stigmatisation in the recent past (and still faces this discrimination to some degree), Matthias's desire for SOCE is not just a simple choice between, say, vanilla and chocolate ice-cream. Put bluntly, they would say that Matthias's desire to have SOCE is a loaded choiceit is a choice that, despite Matthias's intention, expresses to society that being gay is wrong or sinful. Thus, they would say, Matthias's religious values (which support SOCE) are incompatible with mutual respect and therefore not VBP-able.

But is Matthias's desire for SOCE really a loaded decision? Perhaps it is closer to a simple choice than first appears. In an ideal society-a society entirely free of homophobia and heterosexism [2] — choosing to have SOCE would be a 'simple' choice, akin to choosing between ice-cream flavours. It would not be a loaded choice. That is, there is no oppression or stigmatisation in an ideal society, and so opting to have SOCE would not perpetuate oppression or stigma. New Zealand, where Matthias lives, is not an ideal society. Sexual injustice undoubtedly exists. This means that Matthias's desire to have SOCE is not entirely a simple choice. However, New Zealand is a relatively gay-friendly community. The gay community is not as persecuted as it once was. As being gay is becoming increasingly acceptable, perhaps Matthias's desire to have SOCE is becoming closer to a simple preference. If so, then Matthias's desire to have SOCE is more likely to be compatible with mutual respect, and hence, his religious values (which support his desire for SOCE) are VBP-able.

\subsection{Conclusions}

Many readers from the modern Western world might have the intuition that SOCE is unacceptable. This chapter has challenged that intuition by considering the diversity of values involved in considering whether safe, effective and consensual SOCE would be acceptable for someone in Matthias's position.

In VBP, the legitimate values (those that are compatible with mutual respect) of everyone involved must be considered in the decision-making process. The chapter has suggested that only some homophobic values are incompatible with mutual respect. It is possible Matthias's position-he thinks it is okay for people to be gay, but does not want to be gay himself-to be compatible with mutual respect. This is especially so in a relatively gay-friendly society such as New Zealand, as an individual's decision to have SOCE is less likely to express the view that it is not okay to be gay.

It is possible that VBP will come to the conclusion that safe and effective SOCE should be available to Matthias, should he decide this is what he wants. Some might consider this to be a shortfall of VBP. However, recall Matthias's concern that he 
really does want SOCE but has internalised the predominantly gay-friendly views of New Zealand society. VBP provides space to consider this position. In turn, VBP can help to come to a decision that does not ensure the well-being of the gay population at the expense of people like Matthias.

Many questions concerning this case remain unanswered. Is it really the case that Matthias's desire for SOCE is not based on internalised anti-gay sentiments? To what extent should the perspectives of Lachlan, Kirk and the gay community influence Matthias's decision about whether SOCE is right for him? What impact would Matthias having SOCE have on the gay community-would it turn 'Queer villages into ghost towns' [4]? Would Kirk (Matthias's therapist) have an obligation to refer Matthias to someone who practices safe and effective SOCE? It is unlikely to be the case that VBP can definitively answer all these questions. Nevertheless, we should not forget that the power of VBP lies in its recognition of all mutually respectful values.

Acknowledgements Thanks to Andrew Kirby for the use of his very rich case narrative.

\subsection{Guide to Further Sources}

National LGBTQ Task Force. https://www.thetaskforce.org/

Stuff. 2018. "I thought I was a freak": one man's experience with gay conversion therapy. Stuff. 2018. https://www.stuff.co.nz/national/health/105368961/i-thoughti-was-a-freak-one-mans-experience-with-gay-conversion-therapy

Human Rights Watch. 2017 “Have You Considered Your Parents' Happiness?” Conversion Therapy Against LGBT People in China. https://www.hrw.org/ report/2017/11/15/have-you-considered-your-parents-happiness/conversiontherapy-against-lgbt-people

BBC. 2017. China "gay conversion": accounts of shocks and pills. https://www. bbc.com/news/world-europe-41996322

\section{References}

1. Kirby A. Gay-affirmative therapy and emerging integrative solutions. N Z J Counsell. 2008;28(2):37.

2. Delmas C, Aas S. Sexual reorientation in ideal and non-ideal theory. J Polit Philos. 2018;26(4):463-85.

3. Earp BD, Sandberg A, Savulescu J. Brave new love: the threat of high-tech "conversion" therapy and the bio-oppression of sexual minorities. AJOB Neurosci. 2014;5(1):4-12.

4. Behrmann J, Ravitsky V. Turning queer villages into ghost towns: a community perspective on conversion therapies. AJOB Neurosci. 2014;5(1):14-6.

5. Fulford KWM. Values-based practice: fulford's dangerous idea. J Eval Clin Pract. 2013;19(3):537-46. 
6. Kingma E, Banner N. Liberating practice from philosophy - a critical examination of valuesbased practice and its underpinnings. In: Loughlin M, editor. Debates in values-based practice: arguments for and against. Cambridge: Cambridge University Press; 2014. p. 37-49. (Valuesbased practice). https://www.cambridge.org/core/books/debates-in-valuesbased-practice/liberating-practice-from-philosophy-a-critical-examination-of-valuesbased-practice-and-its-under pinnings/8C609F0E1E8FEBCE38E0E0B437BBFC99.

Open Access This chapter is licensed under the terms of the Creative Commons Attribution 4.0 International License (http://creativecommons.org/licenses/by/4.0/), which permits use, sharing, adaptation, distribution and reproduction in any medium or format, as long as you give appropriate credit to the original author(s) and the source, provide a link to the Creative Commons license and indicate if changes were made.

The images or other third party material in this chapter are included in the chapter's Creative Commons license, unless indicated otherwise in a credit line to the material. If material is not included in the chapter's Creative Commons license and your intended use is not permitted by statutory regulation or exceeds the permitted use, you will need to obtain permission directly from the copyright holder. 